Judgment and Decision Making, Vol. 16, No. 2, March 2021, pp. 484-504

\title{
Beyond "fake news": Analytic thinking and the detection of false and hyperpartisan news headlines
}

\author{
Robert M. Ross* David G. Rand ${ }^{\dagger} \quad$ Gordon Pennycook ${ }^{\ddagger}$
}

\begin{abstract}
Why is misleading partisan content believed and shared? An influential account posits that political partisanship pervasively biases reasoning, such that engaging in analytic thinking exacerbates motivated reasoning and, in turn, the acceptance of hyperpartisan content. Alternatively, it may be that susceptibility to hyperpartisan content is explained by a lack of reasoning. Across two studies using different participant pools (total $\mathrm{N}=1,973$ Americans), we had participants assess true, false, and hyperpartisan news headlines taken from social media. We found no evidence that analytic thinking was associated with judging politically consistent hyperpartisan or false headlines to be accurate and unbiased. Instead, analytic thinking was, in most cases, associated with an increased tendency to distinguish true headlines from both false and hyperpartisan headlines (and was never associated with decreased discernment). These results suggest that reasoning typically helps people differentiate between low and high quality political news, rather than facilitate belief in misleading content. Because social media play an important role in the dissemination of misinformation, we also investigated willingness to share headlines on social media. We found a similar pattern whereby analytic thinking was not generally associated with increased willingness to share hyperpartisan or false headlines. Together, these results suggest a positive role for reasoning in resisting misinformation.
\end{abstract}

Keywords: dual-process theory, fake news, misinformation, news media, partisanship

*Department of Psychology, Macquarie University. https://orcid.org/0000-0001-8711-1675. Email: robross46@gmail.com.

${ }^{\dagger}$ Sloan School, and Department of Brain and Cognitive Sciences, Massachusetts Institute of Technology. https://orcid.org/0000-0001-8975-2783.

${ }^{\ddagger}$ Hill/Levene Schools of Business, and Department of Psychology, University of Regina. https://orcid.org/ 0000-0003-1344-6143.

We acknowledge funding from the Ethics and Governance of Artificial Intelligence Initiative of the Miami Foundation (GP, DR), the Social Sciences and Humanities Research Council of Canada (GP), the Canadian Institutes of Health Research (GP), the Luminate Group (GP, DR), the Australian Research Council (RMR), the William and Flora Hewlett Foundation (GP, DR), and the Templeton Foundation (DR).

Copyright: (C) 2021. The authors license this article under the terms of the Creative Commons Attribution 3.0 License. 
Judgment and Decision Making, Vol. 16, No. 2, March 2021 Analytic thinking and news headlines

\section{Introduction}

Social media have become an important source of news for many people (Pew, 2019). Unfortunately, social media can be an outlet for purveyors of misinformation. Moreover, research suggests that false news stories may spread as much (Grinberg, Joseph, Friedland, Swire-Thompson \& Lazer, 2019) or more (Vosoughi, Roy \& Aral, 2018) on social media than news stories that have been fact-checked to be true. This may be problematic, because even a single prior exposure to a false political headline can increase later belief in the headline (Pennycook, Cannon \& Rand, 2018). Although recent analyses indicate that "fake news" is not as prevalent as many thought (and certainly not as prevalent as factual information) (Grinberg et al., 2019; Guess, Nagler \& Tucker, 2019; Guess, Nyhan \& Reifler, 2020), false content is likely to have an impact on individual beliefs (Guess, Lockett, et al., 2020) - for example, the "Pizzagate" incident (Hsu, 2017, June 13) or false beliefs about Donald Trump winning the 2020 U.S. Presidential Election (Pennycook \& Rand, 2021a). Furthermore, content that is misleading and partisan, but not entirely false/fabricated, is likely to be widespread on social media (Faris et al., 2017). For reasons such as these, researchers are rightfully paying increasing attention to the psychological underpinning of susceptibility to false and misleading content (Levy \& Ross, 2021; Pennycook \& Rand, 2021b).

\subsection{Defining the problem}

Since false and misleading content can be loosely classified into a number of categories, we begin by briefly defining a few key terms. Misinformation is false, inaccurate, or misleading information (Wardle, 2018). A particularly flagrant form of misinformation is fake news, which refers to blatantly fabricated information that mimics online news media in form but not in content and can be political or non-political (Lazer et al., 2018). A more subtle form of political misinformation is hyperpartisan news, which is misleading coverage of events that did actually occur with a strong partisan bias (Pennycook \& Rand, 2019b). Although fact-checking approaches tend to focus on outright falsehood (of which fake news is an example), hyperpartisan content is surely more common (Bradshaw, Howard, Kollanyi \& Neudert, 2020; Faris et al., 2017). Naturally, a clear line between "biased" and "unbiased" is difficult to draw, but our focus here is on news content that has a clear and explicit political aim and is either aligned with Democrats or with Republicans (who generally voted for Clinton or Trump, respectively, in the U.S. election of 2016). For simplicity, we will use the term "misleading" when referring to both categories here. Furthermore, while susceptibility to misleading content can manifest in multiple ways, we will focus on believing the content and/or being willing to share it on social media. 
Judgment and Decision Making, Vol. 16, No. 2, March 2021 Analytic thinking and news headlines

\subsection{Theoretical background}

What leads people to be susceptible to misleading content online? One approach is to take a cognitive science lens to the problem and consider, in particular, the role of reasoning (Levy \& Ross, 2021; Pennycook \& Rand, 2021b). Past work has operated under the framework of dual-process theory, which distinguishes between two types of cognitive processes (Evans \& Stanovich, 2013; Kahneman, 2011; Pennycook, Fugelsang \& Koehler, 2015b): System 1 "intuitive" processes that do not require working memory, and are often fast and automatic; and System 2 "analytic" processes that require working memory, and are typically slow and deliberative. However, the relationship between System 2 thinking and susceptibility to misinformation is contested.

A common idea in the dual-process theory literature is that one of the most important functions of analytic processing is the correction (or, possibly, prevention) of false intuitions (Evans \& Frankish, 2009; Stanovich, 2004). This perspective is consistent with classical conceptions of reasoning processes as being directed toward supporting sound judgment and accuracy (e.g., Kohlberg, 1969; Piaget, 1932). When applied to fake and hyperpartisan news content, the implication of this perspective is straightforward: Engaging in System 2 (analytic) processing supports the accurate rejection of misleading content and helps individuals discern between what is true and false. According to this account - which we will refer to here as the "classical reasoning account" - people believe misleading news when they fail to sufficiently engage deliberative (System 2) reasoning processes (Bago, Rand \& Pennycook, 2020; Pennycook \& Rand, 2019c). Furthermore, the reason why misleading content is believed relates to its intuitive appeal - content that is highly emotional (Martel, Pennycook \& Rand, 2020), that provokes moral outrage (Brady, Gantmam \& Van Bavel, 2020; Crockett, 2017), or that draws people's attention; since our cognitive system prioritizes miserly processing (Fisk \& Taylor, 1984; Stanovich, 2004), many individuals fail to effectively stop and reflect on their faulty intuitions. Indeed, it may be that social media are particularly conducive to inattention (Weng, Flammini, Vespignani \& Menczer, 2012) and they may evoke social motivations (e.g., maximize getting "likes") that distract from common accuracy motivations (Pennycook et al., 2021; Pennycook, McPhetres, Zhang, Lu \& Rand, 2020).

The classical reasoning account conflicts starkly with alternatives that focus more strongly on political identity and motivated reasoning. In particular, the "motivated System 2 reasoning" account (henceforth, MS2R ${ }^{1}$ ) argues that people selectively believe factual information that protects their cultural (often political) commitments, and that this selective belief is actually facilitated by deliberative (System 2) thinking processes (Kahan, 2013, 2017; Kahan et al., 2017). This MS2R account has implications opposite to those of the classical reasoning account, which has gained prominence in primarily non-political con-

\footnotetext{
${ }^{1}$ The term "motivated reasoning" has been used to refer to a wide variety of psychological processes (e.g., Kunda, 1990). Here, we refer to the more specific "motivated system 2 reasoning" account that focuses on the impact of deliberation on political polarization (Kahan, 2013, 2017; Kahan, Peters, Dawson \& Slovic, 2017).
} 
Judgment and Decision Making, Vol. 16, No. 2, March 2021 Analytic thinking and news headlines

texts: Whereas MS2R argues that explicit reasoning typically facilitates politically biased information processing (Kahan, 2017), the classical reasoning account argues that explicit reasoning typically facilitates accurate belief formation (Pennycook \& Rand, 2019c).

\subsection{Classical versus motivated reasoning}

Some prior work helps to mediate between the classical and MS2R accounts; although, as we will discuss, the debate is far from settled. In a pair of studies, Pennycook and Rand (2019c) tested the two accounts in the context of political fake news. The MS2R account predicts that people who are more prone (and better able) to engage in deliberation should be more likely to use their cognitive sophistication to protect their prior beliefs and ideological identity. Therefore, more deliberation should be associated with increased belief in political content that is congenial with one's partisan identity, regardless of whether it is fake or real (false or true). Pennycook and Rand (2019c), in contrast, found that people who are more likely and better able to engage in analytic (System 2) reasoning (measured using the Cognitive Reflection Test, CRT; Frederick, 2005) were actually less likely to believe fake news regardless of whether or not it was aligned with their political ideology. Indeed, analytic thinking was associated with being better able to discriminate true and fake news headlines (see also Bronstein et al., 2019; Pehlivanoglu et al., 2020; Pennycook $\&$ Rand, 2020). This result supports the classical reasoning account because it indicates that people who engage in more (and/or better) reasoning are more likely to accurately reject false partisan content and, therefore, are not more likely to engage in politically motivated System 2 reasoning. Furthermore, higher CRT individuals are more likely to accept corrections of false articles that they had previously indicated they were willing to share on social media (Martel, Mosleh \& Rand, 2021). Also consistent with the classical reasoning account, impeding deliberation with cognitive load and time pressure (Bago et al., 2020) or an instruction to rely on emotion (Martel et al., 2020) reduces discrimination by increasing belief in fake news headlines - regardless of the headlines' political alignment.

This prior work paints a fairly clear picture in the context of political fake news, but it represents a somewhat limited test of the motivated versus classical reasoning accounts in the context of misinformation - as fake news is only one part of the misinformation problem. In fact, as mentioned above, recent analyses indicate that fake news was not particularly prevalent during the 2016 U.S. Presidential Election (Grinberg et al., 2019; Guess et al., 2019), perhaps because fake news is often blatantly implausible (Pennycook $\&$ Rand, 2019c). This poses an issue for existing work because motivated reasoning may be limited to cases where the falsehood is not so obvious or blatant, allowing more "intellectual wiggle room" in which politically motivated reasoning can operate. In contrast to fake news, hyperpartisan news is much more prevalent (Faris et al., 2017) and is not so implausible, offering a more relevant and powerful test of the motivated versus classical reasoning accounts. Thus, in this study, we ask participants to make judgments about fake 
Judgment and Decision Making, Vol. 16, No. 2, March 2021 Analytic thinking and news headlines

and hyperpartisan news (in addition to true or "real" news from legitimate mainstream sources).

Another limitation of past work is that it did not tease apart people's judgments about accuracy and their willingness to share. Although Pennycook and Rand (2019c) asked participants to indicate their willingness to share news content on social media, this was done directly after participants made accuracy judgments. This may distort responses because asking people to judge the accuracy of a headline before deciding whether to share it has been shown to dramatically reduce sharing intentions for false headlines (Pennycook, Epstein, et al., 2021). Even judging the accuracy of a single politically neutral headline makes people more discernment in their sharing of true versus fake news (Pennycook, Epstein, et al., 2021; Pennycook, McPhetres, et al., 2020). Thus, in the present study, we randomly assign participants to conditions in which they are asked to rate either the accuracy of headlines or indicate their willingness to share the headlines on social media, allowing a cleaner test of the role of reasoning in sharing decisions.

To summarize, we extend earlier research by examining not only fake news, but also hyperpartisan news; and by separately examining accuracy and sharing judgments. The primary goal of this investigation is to ascertain whether the classical reasoning account or MS2R account explains more variance in how people make judgments about accuracy of news content. To do this, we measure individual differences in analytic thinking via performance on the CRT and relate this to participants' judgments about political news headlines. If analytic thinking supports (and exacerbates) motivated reasoning about biased or misleading information - as per the MS2R account - CRT performance should be positively associated with believing false or misleading news that aligns with political identity (and negatively associated for misaligned false or misleading news). By contrast, if analytic thinking has a general propensity to facilitate accurate beliefs - as per the classical reasoning account - then CRT performance should be negatively associated with believing false or misleading news regardless of political alignment. Furthermore, the classical reasoning account predicts that analytic thinking will be associated with stronger media truth discernment (i.e., higher accuracy judgments for true news relative to hyperpartisan and false news). Finally, a secondary goal of this study is to examine the relationship between CRT performance and willingness to share true, hyperpartisan, and false news because an accuracy motive might not be at the top of people's minds when making decisions about what news to share (Pennycook, Epstein, et al., 2021; Pennycook, McPhetres, et al., 2020). Thus, although willingness to share headlines does not offer a straightforward test of the competing hypotheses, it is of practical relevance to know if analytic thinking is associated with sharing behaviour (indeed, a study using sharing behaviour on Twitter found that people who score higher on the CRT share content from higher quality sources; Mosleh, Pennycook, Arechar \& Rand, 2021). 
Judgment and Decision Making, Vol. 16, No. 2, March 2021 Analytic thinking and news headlines

\section{Method}

We ran two studies which differed only in how participants were recruited. Therefore, for clarity of exposition, we report the methods and results for these studies together as two samples. We preregistered our study and report our target sample sizes, data exclusions, primary analyses, and all measures in the study. Sample sizes were set to be 1000 participants per sample. We arrived at this number by considering the maximum amount of money we wanted to spend on this project and the range of effect sizes found in a related study (Pennycook \& Rand, 2019c). Data, analysis code, survey materials, preregistrations, and additional analyses are available online: https://osf.io/c287t.

\subsection{Participants}

Our first sample was 1000 American participants recruited using Amazon Mechanical Turk (Horton, Rand \& Zeckhauser, 2011). Mechanical Turk users were eligible to participate in our study if their location was USA and they had a HIT approval rate of at least 90. Participants were paid US\$1.30. In total, 1066 participants completed some portion of the study, and we had complete data for 996 participants. The final sample (Mean age = 34.80) included 560 males, 436 females, and 0 other. This study was run June 1st-4th, 2018 (i.e., after the 2016 election for President (Clinton vs. Trump) and congress, and before the November 2018 "midterm" election for congress).

Our second sample was 1000 American participants recruited using Lucid, an online recruiting source that aggregates survey respondents from many respondent providers (Coppock \& McClellan, 2019). Lucid uses quota sampling to provide a sample that matches the national distribution on age, gender, ethnicity and geographic region. Participants are compensated in a variety of ways that include cash and various points programs. In total, 1384 participants completed some portion of the study, and we had complete data for 977 participants. The final sample (Mean age $=45.39$ ) included 473 males, 504 females, and 0 other. This study was run June 12th-14th, 2018.

We compiled a list of false, hyperpartisan, and true news headlines. These headlines were presented in the format of Facebook posts: a picture accompanied by a headline and byline. We removed the source (e.g., "thelastlineofdefense.org") in order to examine responses independently of familiarity with different news sources (we note, however, that manipulating the salience of the source appears to have little influence on whether people judge headlines to be true or false; Dias, Pennycook \& Rand, 2020; Pennycook \& Rand, 2020). Following previous work (e.g., Pennycook \& Rand, 2019c), false news headlines were selected from well-known fact-checking websites (Snopes.com, Politifact.com, and Factcheck.org) and true news headlines were selected from mainstream news sources (e.g., NPR, New York Times). Hyperpartisan news headlines were selected from webpages that were categorised as hyperpartisan by experts (see Pennycook \& Rand, 2019b; e.g., Dailykos.com, breitbart.com). We chose hyperpartisan headlines from across the political 
Judgment and Decision Making, Vol. 16, No. 2, March 2021 Analytic thinking and news headlines

divide that reported actual events but in a biased or misleading manner. For example, one of our headlines was "Trump Says Stupid Things On Fox, Within 2 Hours Prosecutors Use It Against Him In Court." This headline refers to an actual event: Donald Trump admitted that his former lawyer Michael Cohen had previously worked for him to protect him from accusations that he had had an affair with Stormy Daniels. Nonetheless, while this headline refers to an actual event, it does not summarise what Donald Trump actually said or provide useful context, but merely declares that what he said was "stupid".

In all cases, we presented participants with headlines that had a partisan slant: they were either pro-Democrat or pro-Republican. To validate this sorting of items, we conducted a pretest $(\mathrm{N}=467$ ) where MTurk participants were presented a large set of false, hyperpartisan, and true political news headlines. Headlines were selected by the first author, and all three authors discussed which to retain for pre-testing. (These discussions were informal and no quantitative test of inter-rater reliability analysis was performed.) The full set of headlines consisted of 40 items from each category, although each participant rated only 20 randomly selected items in total. Participants were asked to answer four questions for each presented headline (in the following order): 1) "What is the likelihood that the above headline is true?" (on a 7-point scale from "extremely unlikely" to "extremely likely"); 2) "Assuming the above headline is entirely accurate, how favourable would it be to Democrats versus Republicans?" (on a 5-point scale from "more favourable to Democrats" to "more favourable to Republicans"); 3) "Are you familiar with the above headline (have your seen or heard about it before)? (with three response options "yes", "unsure" and "no"); and 4) "In your opinion, is the above headline funny, amusing, or entertaining?" (on a 7-point scale from "extremely unfunny" to "extremely funny"). We then selected five items of each type that were equally different from the scale mid-point for the party favourability question (ProDemocrat: false $=1.14$, hyperpartisan $=1.14$, true $=1.14$; Pro-Republican: false $=1.14$, hyperpartisan $=1.14$, true $=1.13$ ), meaning that the Pro-Democrat items were as favourable for the Democrat Party as the Pro-Republican items were favourable for the Republican Party, both across and within item type.

We measured analytic thinking by summing together the number of correct response to a reworded version of the original three-item CRT (Shenhav, Rand \& Greene, 2012) and a non-numerical four-item CRT (Thomson \& Oppenheimer, 2016). The CRT has been shown to predict diverse psychological outcomes including epistemically suspect beliefs (Pennycook, Fugelsang \& Koehler, 2015a) and to retain its predictive validity across time (Stagnaro, Pennycook \& Rand, 2018) and after multiple exposures (Bialek \& Pennycook, 2018). The full seven-item CRT had acceptable reliability (MTurk: Cronbach's $\alpha=.80$; Lucid: $\alpha=.69$ ).

To code Democrat versus Republican partisanship we asked participants, "Which of the following best describes your political preference?" with six response options: "strongly Democratic", "Democratic", "lean Democratic", "lean Republican", "Republi- 
Judgment and Decision Making, Vol. 16, No. 2, March 2021 Analytic thinking and news headlines

can", "strongly Republican". ${ }^{2}$ Reponses were used to sort participants into two partisan groups: Democrat and Republican.

To code a preference for Hillary Clinton versus Donald Trump we asked participants, "If you absolutely had to choose between only Clinton and Trump, who would you prefer to be the President of the United States?" and offered two response options: "Hillary Clinton" and "Donald Trump".

To identify participants who share on social media we asked, "Would you ever consider sharing something political on social media (such as Facebook and Twitter)?" and offered three response options: "yes", "no," and "I don't use social media accounts". Following previous research (Pennycook, Bear, Collins \& Rand, 2020; Pennycook \& Rand, 2019c) and as per our preregistration, participants who did not answer "yes" to this question had their data excluded from analyses that involved sharing as they do not allow us to cleanly examine the relationship between reasoning and the political content that people are willing to share (i.e., they may not discern between high and low quality content because they are unwilling to share any political content on social media).

\subsection{Procedure}

At the beginning of the survey participants were asked "Do you have a Facebook account?" and "Do you have a Twitter account?". If they answered "no" to both questions they were sent to a debriefing screen and were not permitted to participate in the study. Next, participants were presented with 30 headlines ( 3 x 2 design with 10 of each veracity type $\mathrm{x}$ 15 Pro-Republican and 15 Pro-Democrat) with the order of headline randomized for each participant. Crucially, participants were randomly assigned to the accuracy condition or the sharing condition. In the accuracy condition, participants were asked to judge whether the headline was accurate and unbiased: "Do you think this headline describes an event that actually happened in an accurate and unbiased way?" (response options: "yes" and "no"). In the sharing condition, participants were asked to judge whether they would consider sharing the headline on social media: "Would you consider sharing this story online (for example, through Facebook or Twitter)?" (response options: "yes" and "no"). Finally, each participant was randomly assigned to either have the "yes" response option to the left of the "no" response option or vice versa.

Participants then completed the CRT and, afterward, were asked, "Have you seen any of the last seven-word problems before?" (response options: "yes", "maybe", "no"). In the Lucid sample, but not the MTurk sample, participants then completed the Berlin Numeracy Test (Cokely et al., 2012) for exploratory analysis of the extent to which cognitive reflection and "numeracy" predict performance (Pennycook \& Ross, 2016). Next participants were asked demographic questions (age, gender, education, fluency in English), a question about

\footnotetext{
${ }^{2}$ The survey was constructed so that participants had to respond to every question in the survey. However, due to a minor coding error, participants did not have to respond to this particular question. 23 participants ( $1.15 \%$ of the data) did not respond to this question and were removed from all analyses.
} 
Judgment and Decision Making, Vol. 16, No. 2, March 2021 Analytic thinking and news headlines

political party affiliation (response options: Democrat, Republican, Independent, other), a political preference question (see materials section), a social liberal versus social conservative question, an economic liberal versus economic conservative question, a question about who participants voted for in the 2016 U.S. Presidential Election, a question about Clinton versus Trump preference for president of the US (see materials section), a question how whether participant's social circle tended to vote Republican versus Democrat, a question about how participants would vote in US Congressional elections, two questions about political identity, six questions about trust in different sources of information, one question about frequency of use of social media accounts, one question about sharing political content on social media, one question about the importance of only sharing accurate news on social media, a question about belief in God, and a 14-item version of the Need For Cognitive Closure Scale (Kruglanski et al., 2017; Webster \& Kruglanski, 1994).

Finally, participants were asked if they had responded randomly at any point during the study or if they searched the internet for any of the headlines. And they were asked to provide their ZIP code, to estimate how many minutes the survey took them to complete, and to comment on the survey at their discretion (ZIP codes and comments are removed from online datafile to preserve participant anonymity).

\section{Results}

Descriptive statistics are reported in supplementary materials (Tables S1 and S2). Our preregistered analysis plan was to use the Democrat versus Republican partisanship question to operationalize political partisanship (see methods section). However, during peer review it was argued that the Hillary Clinton versus Donald Trump preference question should be used to operationalize political partisanship in the primary analysis (see methods section), given that the items actually used reflected this distinction more than the traditional differences between the two parties). Consequently, for analyses reported in the main text, political partisanship (which we still label as Democrat vs. Republican) is operationalized as a Clinton versus Trump preference. The preregistered analyses that use the Democrat versus Republican partisanship question to operationalize political partisanship are reported in supplementary materials (Tables S3, S4, S5, and S6). Importantly, as reported later, results are remarkably similar across these two approaches to operationalizing partisanship.

\subsection{Accuracy}

Table 1 shows correlations between CRT performance and perceived accuracy of headlines as a function of headline type (false, hyperpartisan, true), political slant (Pro-Democrat, Pro-Republican), and the partisanship of the participant (Democrat, Republican). Crucially, there was no evidence for a positive correlation between CRT and perceived accuracy of politically consistent fake or hyperpartisan news among either Democrats or Republicans in 
Judgment and Decision Making, Vol. 16, No. 2, March 2021 Analytic thinking and news headlines

either sample. This is starkly inconsistent with the MS2R account, which predicts that people higher in analytic thinking should be better able to convince themselves that politically consistent headlines (i.e., Pro-Democrat headlines for Democrats, Pro-Republican headlines for Republicans) are accurate and unbiased. Rather, in most cases, higher CRT people judged fake and hyperpartisan news to be less accurate than lower CRT people. This is consistent with the classical reasoning account. There were, however, some weak relationships: judgments about hyperpartisan headlines that were pro-Democrat were not significantly associated with CRT for Democrats in either sample. Further, CRT was only very weakly (and not significantly) associated with perceived accuracy for false headlines that were Pro-Republican by Republicans in the Lucid sample (contrary to the MTurk sample, and previous work; (Pennycook \& Rand, 2019c).

TABle 1: Correlation (Pearson $r$ ) between Cognitive Reflection Test performance and perceived accuracy as a function of the political slant of the headline (Pro-Democrat vs ProRepublican), the partisanship of the participant (Democrat, Republican) (partisanship is operationalized as a Hillary versus Trump preference for president of the US), and headline type (False vs Hyperpartisan vs True). MTurk sample: Democrat $N=318$; Republican $N=$ 184. Lucid sample: Democrat $N=266$; Republican $N=217$. ${ }^{* * *} p<.001 ;{ }^{* *} p<.01 ;{ }^{*} p<$ .05 .

\begin{tabular}{|c|c|c|c|c|c|c|c|}
\hline \multirow[b]{2}{*}{ Sample } & \multirow[b]{2}{*}{ Partisanship } & \multicolumn{3}{|c|}{ Pro-Democrat slant } & \multicolumn{3}{|c|}{ Pro-Republican slant } \\
\hline & & False & Hyperpartisan & True & False & Hyperpartisan & True \\
\hline \multirow[t]{2}{*}{ MTurk } & Democrat & $-.36^{* * *}$ & -.04 & $.22^{* * *}$ & $-.32^{* * *}$ & $-.26^{* * *}$ & $.24^{* * *}$ \\
\hline & Republican & $-.50^{* * *}$ & $-.32^{* * *}$ & $-.21^{* *}$ & $-.35^{* * *}$ & $-.21^{* *}$ & $.22^{* *}$ \\
\hline \multirow[t]{2}{*}{ Lucid } & Democrat & $-.21^{* * *}$ & -.10 & $.15^{*}$ & $-.20^{* * *}$ & $-.25^{* * *}$ & $.12^{*}$ \\
\hline & Republican & $-.23^{* * *}$ & $-.15^{*}$ & -.07 & -.11 & $-.20^{* *}$ & .06 \\
\hline
\end{tabular}

There were some relationships consistent with the MS2R account for Republicans when considering true news headlines. Specifically, while there was a consistent positive correlation between CRT and judgments of accuracy of true headlines among Democrats in both samples (and regardless of the political slant of the headlines), Republicans produced an inconsistent pattern of results: CRT was positively associated with judgments for ProRepublican true headlines in the MTurk sample, while being negatively associated with judgments for Pro-Democrat true headlines in the MTurk sample. Both of these results were present in the Lucid sample, but they were (like other results in that sample) weak, and not statistically significant. Note also that the CRT correlations with accuracy judgments of false statements for Democracts were about equally negative for Pro-Democrat and Pro-Republican headlines, but for Republicans the correlation was more negative for ProDemocratic headlines than Pro-Republican headlines. The results are consistent with some 
Judgment and Decision Making, Vol. 16, No. 2, March 2021 Analytic thinking and news headlines

degree of bolstering that results from analytic thinking in Republicans. We shall return to these results in the Discussion section.

To more directly assess the association between CRT and the capacity to discern between high quality (true) and low quality (false or hyperpartisan) news, we computed media truth discernment scores for each category (i.e., a true minus false discernment score and true minus hyperpartisan discernment score). ${ }^{3}$ Table 2 shows correlations between CRT performance and discernment for headlines as a function of partisanship and political slant for both samples. Again, there was no evidence for a negative correlation between CRT and media discernment for either sample. Rather, there was a consistent positive association between CRT and the capacity to discern between high and low quality news content, which is consistent with the classical reasoning account. However, the CRT was only weakly and not significantly associated with increased True-Hyperpartisan discernment among Republicans in the Lucid sample.

TABle 2: Correlation (Pearson $r$ ) between Cognitive Reflection Test performance and accuracy discernment as a function of the political slant of the headline (Pro-Democrat vs Pro-Republican), the partisanship of the participant (Democrat, Republican) (partisanship is operationalized as a Hillary versus Trump preference for president of the US), and form of discernment (True-False vs. True-Hyperpartisan). MTurk sample: Democrat $N=318$; Republican $N=184$. Lucid sample: Democrat $N=266$; Republican $N=217$. ${ }^{* * *} \mathrm{p}<.001 ;{ }^{* *} \mathrm{p}$ $<.01 ;{ }^{*} p<.05$.

\begin{tabular}{lllcrcc}
\hline & & \multicolumn{2}{c}{ Pro-Democrat slant } & & \multicolumn{2}{c}{ Pro-Republican slant } \\
\cline { 3 - 3 } Sample & Partisanship & True-False & True-Hyperpartisan & & True-False True-Hyperpartisan \\
\hline \multirow{2}{*}{ MTurk } & Democrat & $.42^{* * *}$ & $.23^{* * *}$ & & $.38^{* * *}$ & $.38^{* * *}$ \\
& Republican & $.32^{* * *}$ & $.14^{*}$ & & $.42^{* * *}$ & $.33^{* * *}$ \\
\multirow{2}{*}{ Lucid } & Democrat & $.30^{* * *}$ & $.27^{* * *}$ & & $.26^{* * *}$ & $.30^{* * *}$ \\
& Republican & $.15^{*}$ & .08 & & $.15^{*}$ & $.25^{* * *}$ \\
\hline
\end{tabular}

\subsection{Willingness to share}

Table 3 shows correlations between CRT performance and willingness to share headlines as a function of headline type (false, hyperpartisan, true), political slant (Pro-Democrat, Pro-Republican), and the partisanship of the participant (Democrat, Republican). While willingness to share social media content does not provide a direct test of the classical reasoning account versus MS2R account, it is interesting to note that the results for willingness to share was broadly similar to the results for judgments of accuracy. In particular,

\footnotetext{
${ }^{3}$ We pre-registered an intention to report hyperpartisan-false discernment scores too. However, on reflection, we realised that these analyses are not informative - neither hyperpartisan news nor false news describes an event in "an accurate and unbiased way" meaning that it is far from clear how participants' hyperpartisan news minus false discernment judgments should be interpreted.
} 
Judgment and Decision Making, Vol. 16, No. 2, March 2021 Analytic thinking and news headlines

there was no evidence for a positive correlation between CRT and willingness to share false or hyperpartisan news. Rather, CRT was consistently negatively correlated with willingness to share false and hyperpartisan news in the MTurk sample, although these negative correlations were much weaker and mostly not significant in the Lucid sample.

TABLE 3: Correlation (Pearson $r$ ) between Cognitive Reflection Test performance and willingness to share as a function of the political slant of the headline (Pro-Democrat vs ProRepublican), the partisanship of the participant (Democrat, Republican) (partisanship is operationalized as a Hillary versus Trump preference for president of the US), and headline type (False vs Hyperpartisan vs. True). Responses for which participants selected the option indicating that they were unwilling to ever share political news on social media were removed from this analysis. MTurk sample: Democrat $N=182$; Republican $N=120$. Lucid sample: Democrat $N=134$; Republican $N=86$. ${ }^{* * *} p<.001 ;{ }^{* *} p<.01 ;{ }^{*} p<.05$.

\begin{tabular}{cllllllll}
\hline & \multicolumn{3}{c}{ Pro-Democrat slant } & & \multicolumn{3}{c}{ Pro-Republican slant } \\
\cline { 2 - 4 } \cline { 7 - 8 } Sample Partisanship & False & Hyperpartisan & True & & False & Hyperpartisan & True \\
\hline \multirow{2}{*}{ MTurk } & Democrat & $-.30^{* * *}$ & $-.24^{* *}$ & -.07 & & $-.40^{* * *}$ & $-.41^{* * *}$ & $-.25^{* * *}$ \\
& Republican & $-.48^{* * *}$ & $-.44^{* * *}$ & $-.47^{* * *}$ & & $-.27^{* *}$ & $-.37^{* * *}$ & $-.22^{*}$ \\
\multirow{2}{*}{ Lucid } & Democrat & $-.19^{*}$ & -.09 & .02 & & $-.25^{* *}$ & $-.30^{* * *}$ & $-.30^{* * *}$ \\
& Republican & $-.24^{*}$ & -.17 & -.24 & & .03 & -.04 & .08 \\
\hline
\end{tabular}

Interestingly, unlike the results for judgments of accuracy, there were no cases where CRT was clearly positively associated with willingness to share true news content. Indeed, CRT was negatively correlated with willingness to share of true Pro-Republican news content for Democrats in both samples and for Republicans on MTurk (but not Republicans on Lucid). Furthermore, CRT was negatively associated with willingness to share true Pro-Democrat news content for Republicans on MTurk (but, again, not for Republicans on Lucid).

To further explore the association between CRT and overall capacity to discern between high quality (true) and low quality (false or hyperpartisan) news in terms of willingness to share, we computed discernment scores for each category (i.e., true minus false and true minus hyperpartisan). Table 4 shows correlations between CRT performance and media sharing discernment for headlines as a function of partisanship and slant for both samples. Again, there was no strong evidence for a negative correlation between CRT and media sharing discernment in any case for either sample. Rather, among Democrats, there was a consistent positive association between CRT and discernment in the MTurk sample, and a positive association for Pro-Democrat news in the Lucid sample. Republicans showed much weaker correlations, in both directions, mostly not significant. 
Judgment and Decision Making, Vol. 16, No. 2, March 2021 Analytic thinking and news headlines

TABle 4: Correlation (Pearson $r$ ) between Cognitive Reflection Test performance and sharing discernment as a function of the political slant of the headline (Pro-Democrat vs ProRepublican), the partisanship of the participant (Democrat, Republican) (partisanship is operationalized as a Hillary versus Trump preference for president of the US), and form of discernment (True-False vs True-Hyperpartisan). Responses for which participants selected the option indicating that they were unwilling to ever share political news on social media were removed from this analysis. MTurk sample: Democrat $N=182$; Republican $N=120$. Lucid sample: Democrat $N=134$; Republican $N=86$. ${ }^{* * *} p<.001 ; * * p<.01 ;{ }^{*} p<.05$.

\begin{tabular}{llccccc}
\hline & \multicolumn{2}{c}{ Pro-Democrat slant } & & \multicolumn{2}{c}{ Pro-Republican slant } \\
\cline { 2 - 3 } \cline { 5 - 6 } Sample Partisanship & True-False & True-Hyperpartisan & & True-False True-Hyperpartisan \\
\hline \multirow{2}{*}{ MTurk } & Democrat & $.21^{* *}$ & $.24^{* *}$ & & $.16^{*}$ & $.17^{*}$ \\
& Republican & .00 & -.07 & & .06 & $.19^{*}$ \\
\multirow{2}{*}{ Lucid } & Democrat & $.24^{* *}$ & $.18^{*}$ & & -.07 & .00 \\
& Republican & -.01 & -.13 & & .07 & .16 \\
\hline
\end{tabular}

Additional analyses are reported in supplementary materials, including pre-registered analyses that compared accuracy judgments to willingness to share judgments (Tables S7 and S8), a series of pre-registered robustness checks (Tables S9-S16), exploratory robustness checks (Tables S17-S21 and Figure S1), and exploratory investigations of itemlevel correlations between CRT scores and accuracy and willingness to share judgements for each headline (Figures S2-S5).

\section{Discussion}

Across two samples with a total of 1,973 participants, we examined the association between analytic thinking and susceptibility to politically slanted misinformation. In earlier research, the only politically slanted misinformation that was examined was false news (e.g., (Pennycook \& Rand, 2019c), which left questions about other forms of misinformation unaddressed. In the present study we extended this work by also investigating hyperpartisan news. We found essentially no evidence for a positive relationship between analytic thinking and judging politically consistent hyperpartisan or false news headlines to be more accurate and unbiased, which does not support the idea that explicit reasoning is used in a politically motivated way (and, hence, inconsistent with the MS2R account; Kahan, 2017). Instead, we often found a negative relationship. Likewise, we found no evidence for a negative relationship between analytic thinking and discernment between true and false or hyperpartisan news headlines (regardless of political consistency), and in almost all cases we found a positive relationship. Together, these results support the claim that, overall, analytic thinking is directed more at forming accurate beliefs than reinforcing political identity and partisan motivations (Pennycook \& Rand, 2019c). 
Judgment and Decision Making, Vol. 16, No. 2, March 2021 Analytic thinking and news headlines

The second sample exhibited fewer associations that support the classical reasoning account than the first sample. This is noteworthy because the second sample was provided by Lucid, a service that uses quota sampling and matches the national distribution on age, gender, ethnicity and geographic region, while the first sample was provided MTurk which does not quota sample. In addition to demographic differences, however, there was also much less variance in CRT scores on Lucid (where participants performed much worse) - which might also explain the weaker effects. Further work is needed to examine the demographics of participants who show - or fail to show - the associations predicted by the classical reasoning account (Pennycook \& Rand, 2019c).

The differences observed in the results for Democrats and Republicans - in particular, that analytic thinking among Republicans was negatively associated with judging Democratleaning true news to be more accurate in the MTurk sample - merit further investigation. Note that parallel results were observed for false news: the correlation of analytic thinking with accuracy judgments of false news was more negative for Democrat-leaning false news than for Republican-leaning false news among Republicans, but Democrats did not show a difference. (The same quantitative pattern is weakly present in the sharing of news, shown in Table 3, but only in the degree of differences of correlations and not in the direction of the correlations.) These results are not inconsistent with the MS2R hypothesis, although only for Republicans.

We can think of at least three candidate explanations (which are not inconsistent with each other) for these apparent partisan differences. First, the results could depend on an idiosyncratic choice of headlines; in fact, an earlier study using a closely related paradigm did not observe such an asymmetry (Pennycook \& Rand, 2019c). However, correlations between CRT and individual headlines showed that the present pattern of results was generally consistent across all items (supplementary materials Figures S2-S5).

Second, highly analytic conservatives (i.e., Republicans) may have a greater propensity to engage in motivated reasoning than liberals (i.e., Democrats) (Jost, 2017; Jost, van der Linden, Panagopoulos \& Hardin, 2018). A general partisan asymmetry has been found in research on belief in conspiracy theories (van der Linden, Panagopoulos, Azevedo \& Jost, 2021).

Third, heterogeneity of political ideology within the Democrat and Republican groups might lump psychologically different groups together. In particular, it has been argued that at least two dimensions - economic and social ideology — are needed to understand political ideology (Feldman \& Johnston, 2014). People who self-identify as libertarian (an identity that tends to express conservativism on economic issues and liberalism on social issues) usually vote Republican, and there is evidence that libertarians perform better on the CRT than liberals or conservatives (Pennycook \& Rand, 2019a; Yilmaz, Adil Saribay $\&$ Iyer, 2020). Consequently, combining libertarians (even those who ended up favouring Trump) with more socially conservative Republicans might confound analyses. However, this explanation by itself is not sufficient to account for the asymmetry we observed, since 
Judgment and Decision Making, Vol. 16, No. 2, March 2021 Analytic thinking and news headlines

it would also require that libertarians engage in motivated bolstering more than other Republicans. We did not plan the experiment to look for these partisan differences, so a more direct test would be warranted.

Given that there were some cases where performance on the Cognitive Reflection Test did not predict better judgment - for example, most of the accuracy discernment comparisons among Republicans on Lucid - one possible argument is that these results are also consistent with a milder form of motivated reasoning. Specifically, political motivations may be the reason why analytic thinking failed to improve people's judgments (although these differences may also be driven by factors other than political motivations, such as differing factual prior beliefs, as per Tappin et al., 2020a, 2020b). Setting aside the difficulty in interpreting small effects, it would be unreasonable to conclude, based on our data, that reasoning is unaffected by political (or otherwise) motivations. Rather, our data primarily signal that, on balance, reasoning helps more than it hurts when it comes to news headline evaluation. It remains possible, if not likely, that reasoning is sometimes rendered ineffective because of political motivations (or inaccurate priors) in some contexts.

While sharing of social media content does not provide a direct test of the classical reasoning versus MS2R accounts, it is interesting to compare patterns of willingness to share misinformation from the present study to existing research. Earlier research employing a closely related paradigm asked participants if they were willing to share a headline immediately after asking them whether they thought the headline was true (Pennycook \& Rand, 2019c). A limitation of that approach is that questioning participants about the truth of headlines may have influenced their responses about willingness to share headlines (Fazio, 2020; Pennycook, Epstein, al., 2021; Pennycook, McPhetres, et al., 2020). In the present study, questions about accuracy of headlines and willingness to share them were shown to different participants. This separation eliminates the potential source of bias. We found no evidence that analytic thinking predicted a greater willingness to share politically consistent hyperpartisan or false news headlines. By contrast, we often found that analytic thinking was associated with not being willing to share politically consistent false and hyperpartisan news headlines (and never found a significant positive association with willingness to share). These results are also in line with a recent study of Twitter users which found that higher CRT users were more likely to share news on Twitter from outlets that were deemed to be more trustworthy by fact-checkers (Mosleh et al., 2021). A limitation of the present study is that we examined self-reported willingness to share, rather than actual sharing of headlines. Nonetheless, recent research has found self-reported willingness to share of social media predicts actual sharing behaviour on social media (Mosleh, Pennycook \& Rand, 2020).

The present study drew participants from two different American subject pools, and the results are broadly consistently with earlier studies using an partially overlapping, yet substantially different, set of headlines (Pennycook \& Rand, 2019c). Consequently, we would expect that the results of this research will generalize to other American samples and political headlines. Nonetheless, the extent to which these results will show cross-cultural 
Judgment and Decision Making, Vol. 16, No. 2, March 2021 Analytic thinking and news headlines

generalizability is an open question and an important direction for future research. Moreover, at the level of individual headlines with the same political slant, the correlations between CRT and perceived accuracy, and CRT and willingness to share shows some variation (see supplementary materials Figures S2-S5), which suggests that a useful direction for future research could be to examine properties of headlines that influence the magnitude of these correlations.

In summary, earlier studies had examined the relationship between analytic thinking and assessments of fake news. In the present study, we extended this work by examining another form of misinformation: hyperpartisan news. Contrary to the MS2R account, we found little evidence consistent with people using their analytic thinking ability to maintain a belief in fake or hyperpartisan news that supports their political identity. Instead, we found that analytic thinking ability is typically associated with the rejection of misinformation, largely irrespective of ideological alignment.

\section{References}

Bago, B., Rand, D. G., \& Pennycook, G. (2020). Fake news, fast and slow: Deliberation reduces belief in false (but not true) news headlines. Journal of Experimental Psychology: General, 149(8), 1608-1613. http://dx.doi.org/10.1037/xge0000729.

Bialek, M., \& Pennycook, G. (2018). The cognitive reflection test is robust to multiple exposures. Behavior Research Methods, 50(5), 1953-1959. http://dx.doi.org/10.3758/ s13428-017-0963-x.

Bradshaw, S., Howard, P. N., Kollanyi, B., \& Neudert, L.-M. (2020). Sourcing and automation of political news and information over social media in the United States, 20162018. Political Communication, 37(2), 173-193. http://dx.doi.org/10.1080/10584609. 2019.1663322.

Brady, W. J., Gantmam, A. P., \& Van Bavel, J. J. (2020). Attentional capture helps explain why moral and emotional content go viral. Journal of Experimental Psychology: General, 149(4), 746-756. http://dx.doi.org/10.1037/xge0000673.

Bronstein, M. V., Pennycook, G., Bear, A., Rand, D. G., \& Cannon, T. D. (2019). Belief in fake news is associated with delusionality, dogmatism, religious fundamentalism, and reduced analytic thinking. Journal of Applied Research in Memory and Cognition, 8(1), 108-117. http://dx.doi.org/10.1016/j.jarmac.2018.09.005.

Cokely, E. T., Galesic, M., Schulz, E., Ghazal, S., \& Garcia-Retamero, R. (2012). Measuring risk literacy: The Berlin Numeracy Test. Judgment and Decision Making, 7(1), 25-47.

Coppock, A., \& McClellan, O. A. (2019). Validating the demographic, political, psychological, and experimental results obtained from a new source of online survey respondents. Research and Politics, 6(1), 1-15. https://doi.org/10.1177/2053168018822174.

Crockett, M. J. (2017). Moral outrage in the digital age. Nature Human Behaviour, 1(11), 769-771. http://dx.doi.org/10.1038/s41562-017-0213-3. 
Judgment and Decision Making, Vol. 16, No. 2, March 2021 Analytic thinking and news headlines

Dias, N., Pennycook, G., \& Rand, D. G. (2020). Emphasizing publishers does not effectively reduce susceptibility to misinformation on social media. Harvard Kennedy School Misinformation Review, 1(1), 1-12. http://dx.doi.org/10.37016/mr-2020-001.

Evans, J. S. B. T., \& Frankish, K. (Eds.). (2009). In two minds: Dual processes and beyond: Oxford University Press.

Evans, J. S. B. T., \& Stanovich, K. E. (2013). Dual-process theories of higher cognition: advancing the debate. Perspectives on Psychological Science, 8(3), 223-241. http://dx. doi.org/10.1177/1745691612460685.

Faris, R., Roberts, H., Etling, B., Bourassa, N., Zuckerman, E., \& Benkler, Y. (2017). Partisanship, propaganda, and disinformation: Online media and the 2016 U.S. presidential election. The Berkman Klein Center for Internet \& Society Research Publication Series, https://ssrn.com/abstract $=3019414$.

Fazio, L. (2020). Pausing to consider why a headline is true or false can help reduce the sharing of false news. Harvard Kennedy School Misinformation Review. http://dx.doi. org/10.37016/mr-2020-009.

Feldman, S., \& Johnston, C. (2014). Understanding the determinants of political ideology: Implications of structural complexity. Political Psychology, 35(3), 337-358. http://dx. doi.org/10.1111/pops.12055.

Fisk, S. T., \& Taylor, S. E. (1984). Social Cognition: Sage.

Frederick, S. (2005). Cognitive reflection and decision making. Journal of Economic Perspectives, 19(4), 25-42. http://dx.doi.org/10.1257/089533005775196732.

Grinberg, N., Joseph, K., Friedland, L., Swire-Thompson, B., \& Lazer, D. (2019). Fake news on Twitter during the 2016 U.S. presidential election. Science, 363, 374-378. http://dx.doi.org/10.1126/science.aau2706.

Guess, A., Lockett, D., Lyons, B., Montgomery, J. M., Nyhan, B., \& Reifler, J. (2020). "Fake news" may have limited effects on political participation beyond increasing beliefs in false claims. Harvard Kennedy School Misinformation Review, 1(1), 1-12. http://dx. doi.org/10.37016/mr-2020-004.

Guess, A., Nagler, J., \& Tucker, J. (2019). Less than you think: Prevalence and predictors of fake news dissemination on Facebook. Science Advances, 5(eaau4586), 1-8. http:// dx.doi.org/10.1126/sciadv.aau4586.

Guess, A., Nyhan, B., \& Reifler, J. (2020). Exposure to untrustworthy websites in the 2016 US election. Nature Human Behaviour, 4, 472-480. http://dx.doi.org/10.1038/s41562020-0833-X.

Horton, J. J., Rand, D. G., \& Zeckhauser, R. J. (2011). The online laboratory: Conducting experiments in a real labor market. Experimental Economics, 14(3), 399-425. http://dx. doi.org/10.1007/s10683-011-9273-9.

Hsu, S. S. (2017, June 13). 'Pizzagate' gunman says he was foolish, reckless, mistaken and sorry. The Washington Post. Retrieved from https://www.washingtonpost.com/local/ public-safety/pizzagate-shooter-apologizes-in-handwritten-letter-for-his-mistakes-ahead- 
Judgment and Decision Making, Vol. 16, No. 2, March 2021 Analytic thinking and news headlines

of-sentencing/2017/06/13/f35126b6-5086-11e7-be25-3a519335381c_story.html.

Jost, J. T. (2017). Ideological asymmetries and the essence of political psychology. Political Psychology, 38(2), 167-208. http://dx.doi.org/10.1111/pops.12407.

Jost, J. T., van der Linden, S., Panagopoulos, C., \& Hardin, C. D. (2018). Ideological asymmetries in conformity, desire for shared reality, and the spread of misinformation. Current Opinion in Psychology, 23, 77-83. http://dx.doi.org/10.1016/j.copsyc.2018.01. 003 .

Kahan, D. M. (2013). Ideology, motivated reasoning, and cognitive reflection. Judgment and Decision Making, 8(4), 407-424.

Kahan, D. M. (2017). Misconceptions, misinformation, and the logic of identity-protective cognition. Available at SSRN: http://dx.doi.org/10.2139/ssrn.2973067.

Kahan, D. M., Peters, E., Dawson, E. C., \& Slovic, P. (2017). Motivated numeracy and enlightened self-government. Behavioural Public Policy, 1(01), 54-86. http://dx.doi. org/10.1017/bpp.2016.2.

Kahneman, D. (2011). Thinking, fast and slow. New York, NY: Farrar, Straus and Giroux.

Kohlberg, L. (1969). Stage and sequence: The cognitive-developmental approach to socialization. In D. A. Gosling (Ed.), Handbook of Socialization Theory and Research (pp. 347-480): Rand McNally.

Kruglanski, A. W., Gelfand, M. J., Sheveland, A., Babush, M., Hetiarachchi, M., Ng Bonto, M., \& Gunaratna, R. (2017). What a difference two years make: patterns of radicalization in a Philippine jail. Dynamics of Asymmetric Conflict, 9(1-3), 13-36. http://dx.doi.org/ 10.1080/17467586.2016.1198042.

Kunda, Z. (1990). The case for motivated reasoning. Psychological Bulletin, 108(3), 480-498. https://doi.org/10.1037/0033-2909.108.3.480.

Lazer, D. M. J., Baum, M. A., Benkler, Y., Berinsky, A. J., Greenhill, K. M., Menczer, F., . . . Zittrain, J. L. (2018). The science of fake news. Science, 359(6380), 1094-1096. https://doi.org/10.1126/science.aao2998.

Levy, N., \& Ross, R. M. (2021). The cognitive science of fake news. In M. Hannon \& J. de Ridder (Eds.), Routledge Handbook of Political Epistemology, pp. 181-191. Routledge. https://doi.org/10.31234/osf.io/3nuzj.

Martel, C., Mosleh, M., \& Rand, D. G. (2021). You're definitely wrong, maybe: Correction style has minimal effect on corrections of misinformation online. Media and Communication, 9(1), 120-133. https://doi.org/10.17645/mac.v9i1.3519.

Martel, C., Pennycook, G., \& Rand, D. G. (2020). Reliance on emotion promotes belief in fake news. Cognitive Research: Principles and Implications, 5(47). http://dx.doi.org/ 10.1186/s41235-020-00252-3.

Mosleh, M., Pennycook, G., Arechar, A. A., \& Rand, D. G. (2021). Cognitive reflection correlates with behavior on Twitter. Nat Commun, 12(921). https://doi.org/10.1038/ s41467-020-20043-0.

Mosleh, M., Pennycook, G., \& Rand, D. G. (2020). Self-reported willingness to share 
Judgment and Decision Making, Vol. 16, No. 2, March 2021 Analytic thinking and news headlines

political news articles in online surveys correlates with actual sharing on Twitter. PLoS ONE, 15(2), e0228882. http://dx.doi.org/10.1371/journal.pone.0228882.

Pehlivanoglu, D., Lin, T., Deceus, F., Heemskerk, A., Ebner, N. C., \& Cahill, B. S. (2020). The role of analytical reasoning and source credibility on the evaluation of real and fake full-length news articles. https://doi.org/10.31234/osf.io/98zwc.

Pennycook, G., Bear, A., Collins, E. T., \& Rand, D. G. (2020). The Implied Truth Effect: Attaching warnings to a subset of fake news stories increases perceived accuracy of stories without warnings. Management Science, 66(11), 4944-4957. https://doi.org/10. 1287/mnsc.2019.3478.

Pennycook, G., Cannon, T. D., \& Rand, D. G. (2018). Prior exposure increases perceived accuracy of fake news. Journal of Experimental Psychology: General, 147, 1865-1880. https://doi.org/10.1037/xge0000465.

Pennycook, G., Epstein, Z., Mosleh, M., Arechar, A. A., Eckles, D., \& Rand, D. G. (2021). Shifting attention to accuracy can reduce misinformation online. Nature. https://doi.org/ 10.1038/s41586-021-03344-2.

Pennycook, G., Fugelsang, J. A., \& Koehler, D. J. (2015a). Everyday consequences of analytic thinking. Current Directions in Psychological Science, 24(6), 425-432. http:// dx.doi.org/10.1177/0963721415604610.

Pennycook, G., Fugelsang, J. A., \& Koehler, D. J. (2015b). What makes us think? A threestage dual-process model of analytic engagement. Cognitive Psychology, 80, 34-72. http://dx.doi.org/10.1016/j.cogpsych.2015.05.001.

Pennycook, G., McPhetres, J., Zhang, Y., Lu, J. G., \& Rand, D. G. (2020). Fighting COVID19 misinformation on social media: Experimental evidence for a scalable accuracy nudge intervention. Psychological Science, 31(7), 770-780. https://doi.org/10.1177/ 0956797620939054.

Pennycook, G., \& Rand, D. (2021a). Examining false beliefs about voter fraud in the wake of the 2020 Presidential Election. The Harvard Kennedy School Misinformation Review. https://doi.org/10.37016/mr-2020-51.

Pennycook, G., \& Rand, D. G. (2021b). The psychology of fake news. Trends in Cognitive Sciences. https://doi.org/10.1016/j.tics.2021.02.007.

Pennycook, G., \& Rand, D. G. (2019a). Cognitive Reflection and the 2016 U.S. Presidential Election. Personality and Social Psychology Bulletin. https://doi.org/10.1177/ 0146167218783192 .

Pennycook, G., \& Rand, D. G. (2019b). Fighting misinformation on social media using crowdsourced judgments of news source quality. Proceedings of the National Academy of Sciences USA, 116(7), 2521-2526. http://dx.doi.org/10.1073/pnas.1806781116.

Pennycook, G., \& Rand, D. G. (2019c). Lazy, not biased: Susceptibility to partisan fake news is better explained by lack of reasoning than by motivated reasoning. Cognition, 188, 39-50. http://dx.doi.org/10.1016/j.cognition.2018.06.011.

Pennycook, G., \& Rand, D. G. (2020). Who falls for fake news? The roles of bullshit 
Judgment and Decision Making, Vol. 16, No. 2, March 2021 Analytic thinking and news headlines

receptivity, overclaiming, familiarity, and analytic thinking. Journal of Personality, 88, 185-200. http://dx.doi.org/10.1111/jopy.12476.

Pennycook, G., \& Ross, R. M. (2016). Commentary: Cognitive reflection vs. calculation in decision making. Frontiers in Psychology, 7, 1-4. http://dx.doi.org/10.3389/fpsyg. 2016.00009

Pew. (2019). Key findings about the online news landscape in America. https://www. pewresearch.org/fact-tank/2019/09/11/key-findings-about-the-online-news-landscape-inamerica/.

Piaget, J. (1932). The moral judgment of the child: Routledge.

Shenhav, A., Rand, D. G., \& Greene, J. D. (2012). Divine intuition: Cognitive style influences belief in god. Journal of Experimental Psychology: General, 141(3), 423428. http://dx.doi.org/10.1037/a0025391.

Stagnaro, M. N., Pennycook, G., \& Rand, D. G. (2018). Performance on the Cognitive Reflection Test is stable across time. Judgment and Decision Making, 13(3), 260-267.

Stanovich, K. E. (2004). The robot's rebellion: Finding meaning in the age of Darwin: University of Chicago Press.

Tappin, B., Pennycook, G., Rand, D.G. (2020a) Thinking clearly about causal inferences of politically motivated reasoning: Why paradigmatic study designs often prevent causal inference. Current Opinion in Behavioral Sciences 34, 81-87. http://dx.doi.org/10.1037/ xge0000974.

Tappin, B., Pennycook, G., Rand, D.G. (2020b) Rethinking the link between cognitive sophistication and identity-protective bias in political belief formation. Journal of Experimental Psychology: General. http://dx.doi.org/10.1037/xge0000974.

Thomson, K. S., \& Oppenheimer, D. M. (2016). Investigating an alternative form of the Cognitive Reflection Test. Judgment and Decision Making, 11(1), 99-113.

van der Linden, S., Panagopoulos, C., Azevedo, F., \& Jost, J. T. (2021). The paranoid style in American politics revisited: An ideological asymmetry in conspiratorial thinking. Political Psychology, 42(1), 23-51. http://dx.doi.org/10.1111/pops.12681.

Vosoughi, S., Roy, D., \& Aral, S. (2018). The spread of true and false news online. Science, 359, 1146-1151. http://dx.doi.org/10.1126/science.aap9559.

Wardle, C. (2018). Information disorder: The essential glossary. Shorenstein Center on Media, Politics, and Public Policy, Harvard Kennedy School. [Online]. Available: https://firstdraftnews.org/wp-content/uploads/2018/07/infoDisorder_glossary.pdf. [Accessed: 14-Dec-2020].

Webster, D. M., \& Kruglanski, A. W. (1994). Individual differences in Need for Cognitive Closure. Personality Processes and Individual Differences, 67(6), 1049-1062. https:// doi.org/10.1037/0022-3514.67.6.1049.

Weng, L., Flammini, A., Vespignani, A., \& Menczer, F. (2012). Competition among memes in a world with limited attention. Scientific Reports, 2, 335. http://dx.doi.org/10.1038/ srep00335. 
Judgment and Decision Making, Vol. 16, No. 2, March 2021 Analytic thinking and news headlines

Yilmaz, O., Saribay, S. A., \& Iyer, R. (2020). Are neo-liberals more intuitive? Undetected libertarians confound the relation between analytic cognitive style and economic conservatism. Current Psychology, 39, 25-32. http://dx.doi.org/10.1007/s12144-019-0130$\mathrm{X}$. 